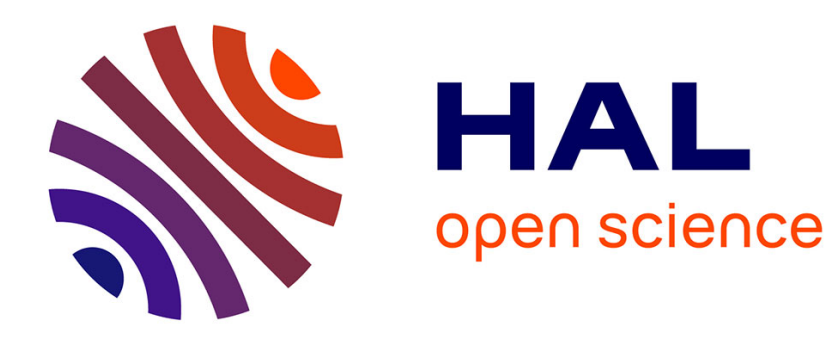

\title{
Photon interactions for electron microscopy applications
}

A. Howie

\section{To cite this version:}

A. Howie. Photon interactions for electron microscopy applications. European Physical Journal: Applied Physics, 2011, 54 (3), 10.1051/epjap/2010100353 . hal-00705152

\section{HAL Id: hal-00705152 \\ https://hal.science/hal-00705152}

Submitted on 7 Jun 2012

HAL is a multi-disciplinary open access archive for the deposit and dissemination of scientific research documents, whether they are published or not. The documents may come from teaching and research institutions in France or abroad, or from public or private research centers.
L'archive ouverte pluridisciplinaire HAL, est destinée au dépôt et à la diffusion de documents scientifiques de niveau recherche, publiés ou non, émanant des établissements d'enseignement et de recherche français ou étrangers, des laboratoires publics ou privés. 


\title{
Photon interactions for electron microscopy applications
}

\author{
A. Howie ${ }^{\mathrm{a}}$ \\ Department of Physics, University of Cambridge, J.J. Thompson Avenue, Cambridge \\ CB3 0HE, UK. \\ Email:ah30@cam.ac.uk
}

The Schrodinger equation is applied to the photon-electron interaction to give a unified picture of the ponderomotive refraction effect, Kapitza-Dirac diffraction as well as near field energy gain and loss processes. Analytical solutions are studied for simple cases and the potential use of these phenomena in applications of electron microscopy is discussed.

\section{Introduction}

Although almost 80 years have elapsed since Kapitza and Dirac pointed out [1] the possibility of an elastic scattering diffraction interaction between an electron and a standing light wave, it is only quite recently that experiments demonstrating and now extending that picture have been conducted. Thanks to the use of lasers in generating the required high light intensity, the diffraction effect has now been observed [2] and its dependence on light intensity studied (for reviews see Batelaan $[3,4]$ ). Closely related to this manifestation of the periodic part of the Kapitza-Dirac potential is the refraction effect produced by its average value known as the ponderomotive potential. Electrons crossing a confined region of high light intensity experience a repulsive potential and consequent phase shift [5]. This simpler effect has yet to be explored in detail, but is the key part of a recent proposal to build a novel device for Zernike phase contrast electron microscopy [6]. The third and most spectacular recent development is the observation of electron energy losses and gains generated at an electron beam that crosses a high intensity light field in the vicinity of nano wires or nanotubes [7]. This experiment was conducted using synchronous femto-second light and electron pulses in the context of a pioneering programme for ultrafast electron microscopy using the pump-probe principle [8]. The effect was predicted [9] and the results obtained [7] have been quantitatively explained by computation of electron's interaction with what were identified as evanescent light waves in the near field region [10].

These developments undoubtedly bring fresh impetus to deeper study of the electronphoton interaction and its potential exploitation in the electron microscope. To supplement image information available from elastic and inelastic electron scattering, routine use is already made of X-ray energy dispersive spectroscopy (XEDS) and cathodoluminescence (CDL) both in scanning electron microscopy (SEM) and in scanning transmission electron microscopy (STEM) [11, 12]. The advantages of photons over electrons for precise spectroscopy and energy-targeted excitation have also led to suggestions for inverse CDL and energy gain experiments [13, 14]. These proposals have not so far been realised but, like XEDS and CDL, rather than depending on a direct 
interaction between photons and fast electrons, would involve an indirect interaction through their mutual involvement in real excitation and de-excitation of electrons in the object studied. The direct interaction that characterizes the recent work just mentioned provides the focus here. A theory covering all three of these recent developments is developed from the Schrodinger equation which is more familiar to electron microscopists than the Dirac eqn. used in [6]. Furthermore, analytical expressions for the energy loss and gain probabilities can be obtained for typical near field situations. These enable the effects of different geometries or changes in other parameters to be swiftly assessed. Finally we discuss the potential usefulness of these developments which are as usual in competition with other approaches.

\section{The Schrodinger semi-classical picture of light interactions}

\subsection{Ponderomotive potential in an optical standing wave}

Using a simple vector potential $\mathbf{A}$ in the Coulomb gauge with $\operatorname{div} \mathbf{A}=0$, the timedependent Schrodinger equation has the form

$$
i \hbar \frac{\partial \Psi}{\partial t}=\frac{1}{2 m}\left[\frac{\hbar}{i} \nabla-e \mathbf{A}\right]^{2} \Psi
$$

With relativistic mass $m=m_{0} / \sqrt{ }\left(1-v^{2} / c^{2}\right)$ and wave vector $k=m / \hbar v=2 \pi / \lambda_{e}$, this equation can be used to describe an electron of velocity $v$, wavelength $\lambda_{e}$ and energy $E=\hbar^{2} k^{2} / 2 m$ moving in an optical field of frequency $\omega$. Effects such as stimulated absorption and emission (but not spontaneous emission, pair production or spin effects) are then included.

We now consider the interaction of such a fast electron travelling in the $\mathrm{z}$ direction with energy $E$ and an electric field $E_{p} \sin (q x) \sin (\omega t)=\left(E_{p} / 2\right)[\cos (q x-\omega t)-\cos (q x+\omega t)]$ corresponding to a standing light wave. The vector potential $\mathbf{A}$ lies in the electric field polarisation direction $\eta(\mathrm{y}$ or $\mathrm{z})$ and has magnitude $\mathrm{A}=\left(\mathrm{E}_{\mathrm{p}} / \omega\right) \sin (\mathrm{qx}) \cos (\omega \mathrm{t})$. Substituting in equation [1] we then find

$$
\begin{aligned}
& i \hbar \frac{\partial \Psi}{\partial t}=\frac{-\hbar^{2}}{2 m} \nabla^{2} \Psi+\frac{e^{2} E_{p}^{2}}{8 m \omega^{2}}[1-\cos (2 q x)] \Psi \\
& +\frac{\hbar e E_{p}}{m \omega} \sin (q x) \cos (\omega t) i \frac{\partial \Psi}{\partial \eta}+\frac{e^{2} E_{p}^{2}}{8 m \omega^{2}}[1-\cos (2 q x)] \cos (2 \omega t) \Psi
\end{aligned}
$$

The second term on the right in the top line of equation (2) can be recognised as the usual time-independent ponderomotive potential. It does not depend on the polarisation of the light and is repulsive in the regions of high light E-field intensity. The remaining two terms would stimulate energy loss and gain processes and, as discussed later below, can in suitable contexts cause real transitions. However in the plane wave situation considered here, they cannot conserve energy and momentum so we ignore them. 


\subsection{Ponderomotive refraction and Kapitza-Dirac scattering}

In the absence of the two time-dependent terms eqn (2) reduces to the stationary Schrodinger equation for $\psi(x, z)=\Psi \exp (i \Omega t)$ describing an electron of energy $E=\hbar \Omega=$ $\hbar^{2} k^{2} / 2 m$ moving in the ponderomotive potential. The behaviour of fast electrons in such periodic potentials is familiar from crystal propagation problems and involves Bragg reflection, channelling, energy band structure etc. At low light intensities we are in the single scattering, kinematical diffraction regime where the electron beam direction is critical for even one of the two possible Bragg reflections $\mathrm{k}->\mathrm{k}+2 \mathrm{q}$ or $\mathrm{k}->\mathrm{k}-2 \mathrm{q}$ to be significantly excited. With increasing light intensity, these two Bragg reflections can be significantly excited simultaneously and we progressively move to a many beam situation involving Bragg reflections of higher and higher order $\pm 2 \mathrm{nq}$. The Bragg angles involved here are however at least three orders of magnitude smaller than those familiar in electron diffraction from crystals. An important consequence then is that, even after quite a long propagation distance $\mathrm{L}$ in the potential, the lateral spreading of the beams $\operatorname{Ln} \theta=2 \mathrm{Lnq} / \mathrm{k}$ may still be very small compared with the "lattice parameter" $\pi / \mathrm{q}$. When all the beams then effectively move in the same direction as the incident electron (along $\mathrm{z}$ for instance with the trajectory e1 in fig. 1), the Schrodinger eqn. has a simple one-dimensional "phase grating" solution which has already been applied in Kapitza-Dirac theory [3].

$$
\begin{aligned}
& \Psi \exp (i \Omega t)=\psi(x, z)=\exp [i k z-i \beta(1-\cos (2 q x)) z] \\
& =\exp (i k z-i \beta z)\left[J_{0}(\beta z)+2 \sum_{n=1}^{\infty} i^{n} J_{n}(\beta z) \cos (2 n q x)\right] \\
& \beta=\frac{e^{2} E_{p}^{2}}{8 k \hbar^{2} \omega^{2}}
\end{aligned}
$$

Here the progressive development with increasing propagation distance $\mathrm{z}$ of higher order Bragg reflections 2nq just discussed is described more precisely by the Bessel function expansion. After a distance $\mathrm{z}=\mathrm{L}$, reflections of order $\mathrm{n}= \pm \beta \mathrm{L}$ will be significantly excited which indicates that the condition for the validity of the phase grating approximation is $2 \beta \mathrm{L}^{2} \mathrm{q}^{2}<<\pi \mathrm{k}$ or $(\mathrm{eE} / \mathrm{h} \omega)^{2}<<4 \pi(\mathrm{k} / \mathrm{qL})^{2}=8 \pi^{3} /\left(\mathrm{qL} \lambda_{\mathrm{e}}\right)^{2}$. Even at the very high peak intensity levels of $10^{15} \mathrm{Wm}^{-2}$ available with pulsed lasers, $(\mathrm{eE} / \mathrm{h} \omega)^{2}$ would be a million times less than this value.

To get a the $-\pi / 2$ phase shift required for phase contrast electron microscopy with a $200 \mathrm{keV}$ electron propagating a distance $L=1 \mu \mathrm{m}$ in a direction where it samples the average ponderomotive potential $\left(\mathrm{eE}_{\mathrm{p}}\right)^{2} / 8 \mathrm{~m} \omega^{2}$ then implies

$$
\frac{e^{2} E_{p}^{2}}{\hbar^{2} \omega^{2}}=\frac{8 \pi^{2}}{\lambda_{e} L} \approx 3.2 \times 10^{19} \mathrm{~m}^{-2}
$$

Choosing a wavelength $\lambda=2 \mu \mathrm{m}$ (i.e. $\hbar \omega=0.56 \mathrm{eV}$ ) for the plane wave of amplitude $\mathrm{E}_{\mathrm{p}} / 2$ incident on the cavity, this corresponds to a very high intensity of $I=c \varepsilon_{0} E_{p}{ }^{2} / 4=6.6 \times 10^{15}$ $\mathrm{Wm}^{-2}$. A similar figure arises in the analysis of Muller et al [5] who suggest various 
ways of using cavities with highly reflecting walls to increase the field from readily obtainable continuous wave lasers. The high intensities required could also be produced by employing pulsed operation for both the optical and the electron beam. In the last few years, Zewail and colleagues have impressively demonstrated precise synchronisation of optical and electron pulses on the near femto-second time scale for ultrafast microscopy as well as stimulated energy loss and gain processes $[7,8]$. The $10^{8} \mathrm{~s}^{-1}$ pulse repetition rate allows a high resolution image to be acquired in a few seconds.

For propagation parallel to the optical nodes and antinodes however equation (4) shows that the phase shift will vary periodically with $\mathrm{x}$, resulting in undesirable oscillations between phase and amplitude contrast across the diffraction plane. This effect would be mitigated for propagation across the curved wave fronts in a spherical or parabolic cavity but, for propagation through less sharply focused standing wave systems, it would be better for the fast electron to travel cross the antinodes at an angle chosen to sample them evenly (as for direction e2 in fig 1).

\section{Stimulated energy loss and gain processes}

\subsection{Phase grating theory for time-dependent potentials}

The phase grating theory employed above can be usefully extended to deal with potential energy loss and gain events like those in equation (2) arising from a time-dependent potential

$$
i \hbar \frac{\partial \Psi}{\partial t}=\frac{-\hbar^{2}}{2 m} \nabla^{2} \Psi+\left[V(\mathbf{r}) e^{-i \omega t}+V^{*}(\mathbf{r}) e^{i \omega t}\right] \Psi
$$

A specific form for the potential $\mathrm{V}(\mathrm{x}, \mathrm{y}, \mathrm{z})$ will be used later but here we assume only that it is small in magnitude compared with the electron energy $E=\hbar \Omega$ and varies extremely slowly with position on the scale of the electron wavelength $\lambda_{\mathrm{e}}=2 \pi / \mathrm{k}(2.5 \mathrm{pm}$ for $\mathrm{E}=$ $200 \mathrm{keV}$ ). For an electron incident in the $\mathrm{z}$ direction we therefore can ignore the $\mathrm{x}$ and $\mathrm{y}$ derivatives of $\Psi$ and look a quasi-one dimensional solution of WKB type analogous to the phase grating solution studied above.

$$
\Psi(\mathbf{r}, t)=\exp \left[i(k z-\Omega t)+i\left(S(\mathbf{r}) e^{-i \omega t}+S^{*}(\mathbf{r}) e^{i \omega t}\right)\right\rfloor
$$

Substituting in eqn (5), identifying the different frequency components and introducing $\mathbf{r}^{\prime}$ with the same $\mathrm{x}$ and $\mathrm{y}$ components as $\mathbf{r}$ but with its own $\mathrm{z}$ component $\mathrm{z}$, we find

$$
\begin{aligned}
& \frac{\partial S(\mathbf{r})}{\partial z}-\frac{i \omega}{v} S(\mathbf{r})=-\frac{m V(\mathbf{r})}{\hbar^{2} k} \\
& S(\mathbf{r})=-\frac{m}{\hbar^{2} k} \exp \left(i \frac{\omega}{v} z\right) \int_{-\infty}^{z} V\left(\mathbf{r}^{\prime}\right) \exp \left(-i \frac{\omega}{v} z^{\prime}\right) d z^{\prime}
\end{aligned}
$$


The integral in eqn (7), describes the energy gain process with appropriate momentum change for the fast electron. For scattering to occur, the potential V must have a Fourier transform with wave vector component along $\mathrm{z}$ of $\omega / \mathrm{v}$ which is impossible in the case of far field light waves whose field has a Fourier transform only at wave vector components $\mathrm{q}_{\mathrm{z}} \leq \omega / \mathrm{c}<\omega / \mathrm{v}$. As will be seen below, scattering is however possible in the near field region of a light scattering or emitting object or in the case of an evanescent light wave. Since the complex conjugate of equation (7) holds for $\mathrm{S}^{*}$ and describes the energy loss process, we can rewrite equation (6) as

$$
\begin{aligned}
& \Psi(\mathbf{r}, t)=\exp \left[i(k z-\Omega t)+i|I(\mathbf{r})| \cos \left(\frac{\omega z}{v}-\omega t+\delta\right)\right] \\
& =\exp [i(k z-\Omega t)]\left[J_{0}(|I(\mathbf{r})|)+2 \sum_{n=1}^{\infty} i^{n} J_{n}(|I(\mathbf{r})|) \cos \left(\frac{n \omega z}{v}-n \omega t+n \delta\right)\right]
\end{aligned}
$$

where $I(\mathbf{r})=|I(\mathbf{r})| \exp (i \delta(\mathbf{r}))=\frac{2 m}{\hbar^{2} k} \int_{-\infty}^{z} V\left(\mathbf{r}^{\prime}\right) \exp \left(\frac{i \omega z^{\prime}}{v}\right) d z^{\prime}$

In eqn (8) the positive integer $n$ is associated with both energy loss and gain $\pm n \hbar \omega$ with appropriate change of the fast electron wave vector in each case The probabilities of a energy loss $n \hbar \omega$ or gain $-n \hbar \omega$ are always the same and equal to $\left[\mathrm{J}_{\mathrm{n}}(|\mathrm{I}(\mathrm{x}, \mathrm{y}, \mathrm{z})|)\right]^{2}$ which for each image point $(\mathrm{x}, \mathrm{y})$ usually first increases with $\mathrm{z}$ but can then decrease either because $|\mathrm{I}|$ decreases or because the loss (or gain) spreads to higher values. This simple analytic behaviour of course depends on the validity of the phase grating approximation. Although the peak heights for gains and losses actually observed [7] do not conform to the simple Bessel function law for a single value of $|\mathrm{I}|$, it should be realised that in practice the spectra are collected from a range of image points and are therefore averaged over a range of values of $|\mathrm{I}|$. We can now apply this theory to a simple example.

\subsection{Cylindrical wave analysis}

As a first step in modelling the radiation from the illuminated silver wire and nanotube studied in [7], we refer to the general expressions given by Stratton [15] for scattered waves emerging from a long, slender cylinder lying in the y direction and illuminated with light at a frequency $\omega=$ qc polarised along $\theta=0$. For the dipole waves associated with azimuthal quantum numbers $m= \pm 1$ the vector potential components $A_{\rho}$ and $A_{\theta}$ and the closely related electric field components $E_{\rho}$ and $E_{\theta}$ in the $\rho-\theta$ (i.e. $x-z$ ) plane are given by

$$
\begin{aligned}
& A_{\rho}(\rho, \theta)=\frac{E_{\rho}(\rho, \theta)}{i \omega}=\frac{E_{s} \cos \theta}{\rho} H_{1}^{(1)}(q \rho) e^{-i \omega t} \\
& A_{\theta}(\rho, \theta)=\frac{E_{\theta}(\rho, \theta)}{i \omega}=-E_{s} \sin \theta \frac{d H_{1}^{(1)}(q \rho)}{d \rho} e^{-i \omega t}
\end{aligned}
$$


Here the amplitude constant $\mathrm{E}_{\mathrm{s}}$ for the scattered field arises from the boundary conditions at the cylinder surface and would depend not only on the incident light amplitude but also on the dielectric response function of the cylinder material with associated resonance frequencies. $\mathrm{H}_{1}{ }^{(1)}=\mathrm{J}_{1}+\mathrm{iY} \mathrm{Y}_{1}$ is the usual Hankel function describing an outgoing wave in the far field where only the $\mathrm{E}_{\theta}$ component survives. To find the potential $\mathrm{V}(\mathbf{r})$ used in equation (5) for interaction with a fast electron moving in the $\mathrm{z}$ direction we require the vector potential component $A_{z}$. In the simplest case, with the polarisation direction $\theta=0$ taken along $\mathrm{x}$ as shown in fig. $2, \mathrm{~A}_{\mathrm{z}}$ is given by the expression

$$
\begin{aligned}
& A_{z}=-A_{\rho} \sin \theta+A_{\theta} \cos \theta \\
& =-\frac{E_{s}}{i \omega} \cos \theta \sin \theta\left[\frac{H_{1}^{(1)}(q \rho)}{\rho}+\frac{d H_{1}^{(1)}(q \rho)}{d \rho}\right] e^{-i \omega t} \\
& V(\mathbf{r})=\frac{\hbar k}{m} e A_{z}=-\frac{\hbar k}{i \omega m} E_{s} F\left(q \sqrt{x^{2}+z^{2}}\right) \frac{x z}{\left(x^{2}+z^{2}\right)^{3 / 2}} \\
& F(\xi)=\left[H_{1}^{(1)}(\xi)+0.5 \xi\left(H_{0}^{(1)}(\xi)-H_{2}^{(1)}(\xi)\right)\right]
\end{aligned}
$$

Finally, to compute the quantity I(r) used in equation (8), it is convenient to resort to dimensionless coordinates $X, Y, Z$ defined by $x / X=y / Y=z / Z=v / \omega$ and also to note that $\mathrm{qv} / \omega=\mathrm{v} / \mathrm{c}$. Provided that the unobstructed electron path length near the cylinder is many times the impact parameter $\mathrm{x}$ we can integrate in equation (9) over the whole range of $\mathrm{z}$ to obtain I which is then just a function of $\mathrm{X}$.

$$
\begin{aligned}
& I(X)=-\frac{4 e E_{s} v}{\hbar \omega^{2}} K(X) \\
& K(X)=i \int_{0}^{\infty} F_{c}\left(\frac{v}{c} \sqrt{X^{2}+Z^{2}}\right) \frac{X Z \sin Z}{\left(X^{2}+Z^{2}\right)^{3 / 2}} d Z
\end{aligned}
$$

As indicated in equation (8) the strength of the energy losses and gains in these two cases is controlled by $|\mathrm{I}(\mathbf{r})|$ and hence in this case $|\mathrm{K}(\mathrm{X})|$ which is plotted in fig $4 .|\mathrm{K}(\mathrm{X})|$ reaches a maximum at $1=X=\omega x / v$ i.e. $x=(v / 2 \pi \mathrm{c}) \lambda \approx 57 \mathrm{~nm}$ for $2.4 \mathrm{eV}$ photons. This lies slightly inside the $150 \mathrm{~nm}$ diameter nanotube studied in [7]. Of course analysis of this section applies only to points outside the cylinder where in this case $|K(\mathbf{X})|$ will be a monotonically decreasing function of impact parameter. As already mentioned, the dependence on $\mathrm{X}$ generally means that the spectral peaks will deviate from the simple Bessel behaviour when collected over a range of $\mathrm{X}$ values.

\subsection{Real cylindrical wave situations}

The ideally simple geometry just assumed for the cylindrical system is scarcely likely to apply exactly in practice. Although only schematic diagrams are available, these indicate that illumination used in $[7,8]$ enters the microscope column in the $\mathrm{x}$ direction but is then 
deflected by a mirror to travel at an angle $\theta_{\mathrm{p}}$ of perhaps $20^{\circ}$ to the electron beam direction $z$. The preceding analysis can easily be extended to cover any value of $\theta_{p}$ and energy losses and gains will still result if the scattered light field has an electric field component along z. An additional complication may arise when the polarization does not lie either in or normal to the $\mathrm{x}-\mathrm{z}$ plane since its direction may then be modified by such a mirror.

Furthermore, our analysis so far assumes that the cylindrical object illuminated has its axis along the $y$-direction normal to the $x-z$ plane jointly defined by the incident photon and electron beams. Provided that the angle of rotation about $\mathrm{z}$ between object and image is known, suitable objects can be selected whose image projections in the $\mathrm{x}-\mathrm{y}$ plane lie make an angle $\phi_{\mathrm{L}}$ with $\mathrm{y}$ which is as small as possible. As indicated in figure 3 , the axis of the chosen cylinder may then lie at some non-zero angle $\theta_{\mathrm{L}}$ to the $\mathrm{x}-\mathrm{y}$ plane with direction cosines $\left\{-\cos \left(\theta_{\mathrm{L}}\right) \sin \left(\phi_{\mathrm{L}}\right), \cos \left(\theta_{\mathrm{L}}\right) \cos \left(\phi_{\mathrm{L}}\right), \sin \left(\theta_{\mathrm{L}}\right)\right\}$. The photon wave vector $\mathrm{q}$ will then have a component parallel to the cylinder axis which will be given by $\mathrm{q}_{\mathrm{L}}=$ $\mathrm{q}\left\{\cos \left(\theta_{\mathrm{L}}\right) \sin \left(\theta_{\mathrm{p}}\right) \sin \left(\phi_{\mathrm{L}}\right)+\sin \left(\theta_{\mathrm{L}}\right) \cos \left(\theta_{\mathrm{p}}\right)\right\}$ and can excite waves with this component. The image actually obtained with y polarization [7] does indeed show weak fringes along the surface of the wire which may depict the excitation of a standing wave with intensity maxima separated by $\pi / \mathrm{q}_{\mathrm{L}} \approx 400 \mathrm{~nm}$. Photons of energy $2.4 \mathrm{eV}$ photons with $\lambda=2 \pi / \mathrm{q}=$ $524 \mathrm{~nm}$ were used so $\mathrm{q}_{\mathrm{L}} / \mathrm{q} \approx 0.67$. Unless the azimuthal misalignment angle $\phi$ was rather large, this would therefore imply a tilt angle $\theta_{\mathrm{L}}$ of about 45 degrees. When waves with a wave vector component $\mathrm{q}_{\mathrm{L}}$ along the wire are excited, the scattered wave vector in the normal to the wire becomes $\sqrt{ }\left(\mathrm{q}^{2}-\mathrm{q}_{\mathrm{L}}^{2}\right)$. The wave becomes evanescent in this direction only for $\mathrm{q}_{\mathrm{L}}>\mathrm{q}$ but such waves cannot be excited by external light illumination.

\section{Discussion of future prospects}

Many thin phase objects of intense interest, particularly in biology, are currently imaged in the electron microscope by defocus phase contrast methods. Electron holography can be an effective alternative solution in some cases, particularly near the edge of a hole in the specimen to allow for transmission of an undisturbed reference beam. In principle a Zernike-style phase shifting plate placed in the back-focal plane could more generally produce phase contrast over a wide range of spatial frequencies but physical plates also scatter the electrons and suffer from a variety of problems, particularly contamination and beam damage. An obstruction-free phase shifting device based purely on the action of external fields in vacuum is therefore attractive. To achieve the necessary light intensity in a region just a few microns in extent with current continuous-wave lasers is very demanding however and will require an efficient optical cavity [5].

Using the much higher light intensities available in pulsed mode operation $[7,8]$ it might be possible to achieve the desired result without any very sophisticated cavity. As already noted, the pulsed mode technology is already successfully employed for high resolution pump-probe electron microscopy and has produced the photon-induced electron energy loss and gain events discussed above. Equation (4) indicates that in these experiments the ponderomotive potential must also give rise to a phase shift $\phi=\mathrm{e}^{2} \mathrm{E}_{\mathrm{p}}{ }^{2} \lambda_{\mathrm{e}} \mathrm{L}$ 
/ $16 \pi$. The $400 \mathrm{fs}$ interaction time indicated in [7] for the photon and electron pulses would correspond for a $200 \mathrm{keV}$ electron to a propagation distance $\mathrm{L}$ of $80 \mu \mathrm{m}$ in the illumination field $\mathrm{E}_{\mathrm{i}}$ with peak radiance $c \varepsilon_{0} \mathrm{E}_{\mathrm{i}}^{2} / 4$ of $10^{15} \mathrm{Wm}^{-2}$. The resulting large phase shift of nearly $6 \pi$ is slowly varying over perhaps an area of diameter $10 \mu \mathrm{m}$ or greater and not readily detectable in the image except possibly at large defocus. Near the nanowire however the phase shift will be locally increased by the presence of the scattered field $E_{s}$ given by eqn. (9). Since $\mathrm{E}_{\mathrm{p}}{ }^{2}=\left(\mathbf{E}_{\mathrm{i}}+\mathbf{E}_{\mathrm{s}}\right)^{2}$ we should strictly add these fields vectorially but for simplicity here we assume that on average they are in quadrature and of equal amplitude so that $E_{p}{ }^{2}$ is locally doubled over a path length $L$ of about $150 \mathrm{~nm}$ equal to the nanotube diameter. The additional phase shift localised near the nanotube is thus estimated as $10^{-2} \pi$. At still higher peak laser power this additional phase local shift could be detected.

Another potential rival for an obstruction-free phase shifter proposed by Rose [16] involves an additional static field stage in the multipolar lens stack now widely used for aberration correction. The required $\pi / 2$ phase shift over the whole $2 \pi$ range of azimuthal angles in the $\mathrm{x}-\mathrm{y}$ plane is applied in two steps of $\pi / 4$ acting over the $\mathrm{x}$ and $\mathrm{y}$ sectors in turn. Both of these schemes are very sophisticated but, if eventually available at reasonable cost and routinely operable, would find a large market.

As a means of accessing the electromagnetic near field region, the electron energy loss and gain processes recently observed [7] offer an intriguing new option in addition to those already being actively pursued through the use of an ultra-sharp tip as in near-field scanning optical microscopy or with a sub-wavelength super-lens [17]. The optical fields required are rather high however and are perhaps only achievable in simple elastic scattering processes with intense laser pulses. In this context it is not clear whether they can supply any significant information about the object not already more simply available from electron microscope imaging in conjunction with normal electron energy loss spectroscopy (EELS). Aloof beam mode EELS depends on a rather similar interaction but the exciting wave comes from the electron itself and is intrinsically evanescent. On small objects such as spheres, radiating waves can thus be excited by a passing electron but, and particularly in closer encounters, not just dipolar waves as in the case of light excitation considered here. On more extended objects the both the exciting and the excited waves associated with a passing electron are evanescent. All of these factors mean that e-beam exploration of the near field or evanescent wave regions can be carried out with higher spatial resolution than will be achieved with the photon interactions considered here. Aloof beam electron excitation also extends over a very wide frequency range so that the full spectral response of the object, including any resonances, is covered in each observation. Tuned laser excitation, becoming more available in pulsed mode, would be needed to achieve a comparable result.

In addition to the exploration of ultrafast processes facilitated by the precise timing capability in the pump probe experiments, the very high light intensities available may open up new spectral opportunities through non-linear sample response. Sum difference spectroscopy for instance depends on the second order response and is sensitive to the surface region because an absence of inversion symmetry is necessary. So far however, 
although applied to assemblies of particles as well as to flat surfaces, it is still a broad beam technique $[18,19]$. A degree of spatial localisation has been achieved in other nonlinear spectroscopies such as coherent anti-Stokes Raman spectroscopy by operating in the near field enhancement region of an AFM tip and using three pulsed input beams of different frequencies [20]. In an electron microscope experiment several input pulses of different frequencies could likewise be used. The electron beam could in principle interact directly only with output beams in their near field region and if any of the nonlinear outputs had sufficient intensity to produce detectable electron energy losses and gains the spatial resolution and scope of these spectroscopies could perhaps be dramatically improved. Once again however it could be more practical to regard the electron beam as an input source either in substitution or addition to the optical inputs already used. Pulsed mode operation and phase sensitive detection would then offer additional advantages. Infrared or Raman spectra of selected nano-scale objects, altered under e-beam excitation, might then be detected in the far-field optical output signals.

\section{Acknowledgements}

I am grateful to Javier Garcia de Abajo, Bob Glaeser, Holger Muller, Bryan Reed and John Spence for very helpful discussions. Shortly after this work was submitted, I became aware that a paper with a considerably more detailed analytical analysis of the energy loss and gain processes had independently been prepared by Ahmed Zewail and his colleagues. I am indebted to him for illuminating discussions and for showing me a first draft of this paper now in submission to New Journal of Physics.

\section{References}

1. P.L. Kapitza and P.A.M. Dirac, Proc. Cam. Phil. Soc., 29, 297 (1933)

2. D.L. Freimund, K. Alfatooni and H. Batelaan, Nature 413, 142 (2001)

3. H. Batelaan, Contemp. Phys. 41, 369 (2000)

4. H. Batelaan, Rev. Mod. Phys. 79, 929 (2007)

5. T.W.B. Kibble, Phys. Rev. Lett. 16, 1054 (1966)

6. H. Muller, Jian Jin, R. Danev, J.Spence, H. Padmore and R.M. Glaeser, New J. Phys., 12, 073011 (2010)

7. B. Barwick, D.J. Flannigan and A.H. Zewail, Nature 462, 902 (2009)

8. A.H. Zewail and J.M. Thomas, 4D Electron Microscopy (Imperial Coll. Press, London, 2010)

9. F.J. Garcia de Abajo and M. Kociak, New J. Phys. 10, 073035 (2008)

10. F.J. Garcia de Abajo, A. Asenjo-Garcia and M. Kociak, Nano Lett., 10, 1859 (2010)

11. S.J. Pennycook, L.M. Brown and A.J. Craven, Phil. Mag. 41, 589 (1980)

12. Y. Yamamoto, K. Araya, F.J. Garcia de Abajo, Phys. Rev. B, 64, 205419 (2007)

13. A. Howie, Inst.of Phys. Conf. Ser., 161, 311 (1999)

14. A. Howie, Microsc. and Microanal., 15, 314 (2009)

15. J.A. Stratton, ElectromagneticTheory, (McGraw-Hill, New York, (1941)

16. H. Rose, Ultramicroscopy, 110, 488 (2010)

17. Z. Liu, S. Durant, H. Lee, Y. Pikus, Y. Xiong, C. Sun and X. Xiang, Optics Expr., 15, 6947 (2007)

18. H. Unterhalt, G. Rupprechter and H.-J. Freund, J. Phys. Chem. B 106, 356 (2002) 
19. O. Pluchery, C. Humbert, M. Valamanesh, E. Lacaze and B. Busson, Phys. Chem. Chem. Phys., 11, 7729 (2009)

20. A. Volkmer, L.D. Book and X.S. Xie, Appl. Phys. Lett., 80, 1505 (2002) 


\section{Figure captions}

Fig. 1. Schematic diagram of electron beam paths near the antinodes in a standing light wave. Such a phase shifting device, placed in the back-focal plane, would not intercept the waves scattered by the object but only the unscattered wave. For aligned paths such as e1, the periodic optical potential would generate Kapitza-Dirac diffraction but this effect would be averaged out for trajectories like e2.

Fig. 2. Illumination arrangement and coordinate set up assumed for the illustrative calculation of photon- electron interaction near a cylinder .

Fig. 3. Depiction of the cylindrical scattering geometry more likely in practical cases.

Fig. 4. Computed results for $|\mathrm{K}(\mathrm{X})|$ given by equation (11). 
Figure 1

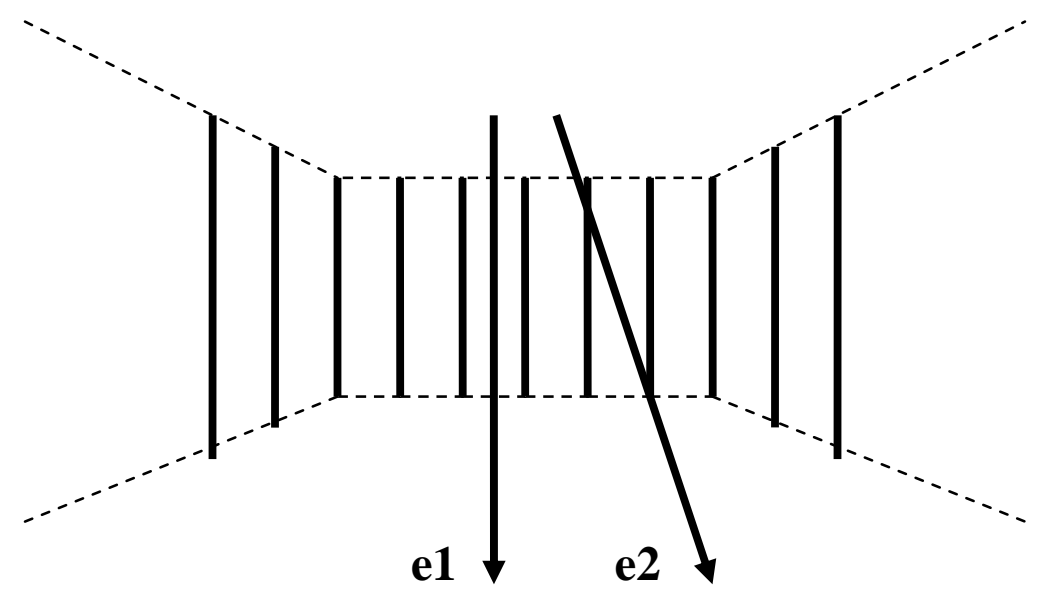


Figure 2

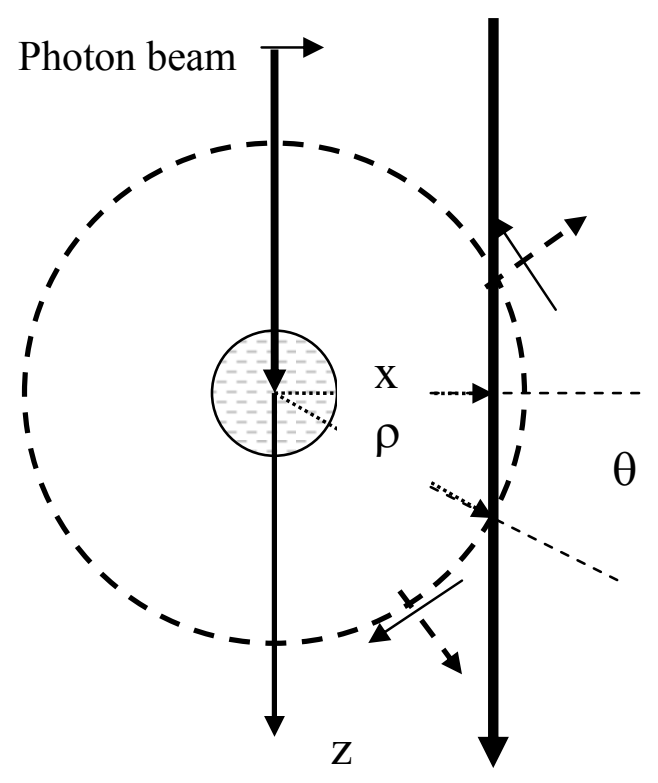


Figure 3

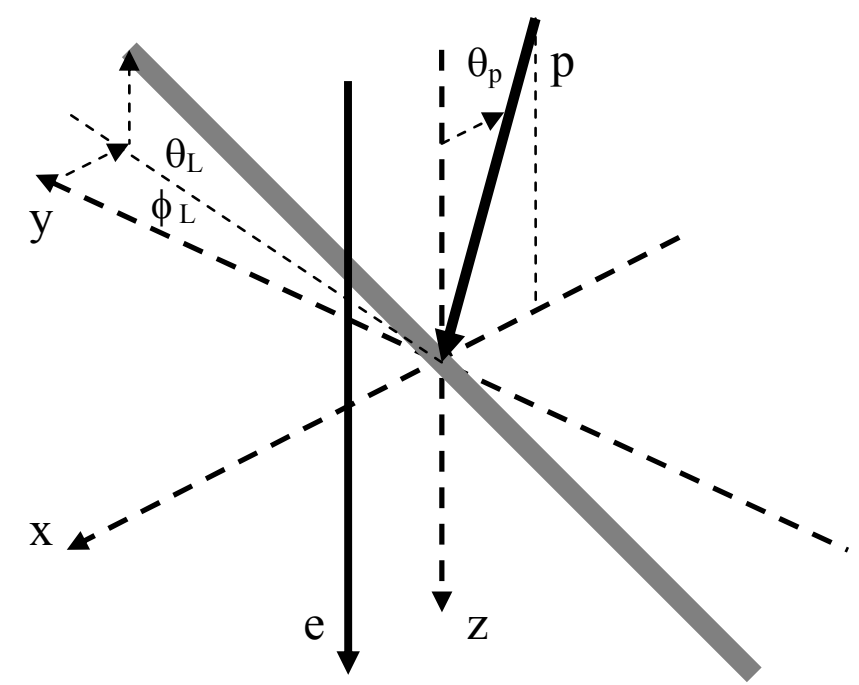

14 
Figure 4

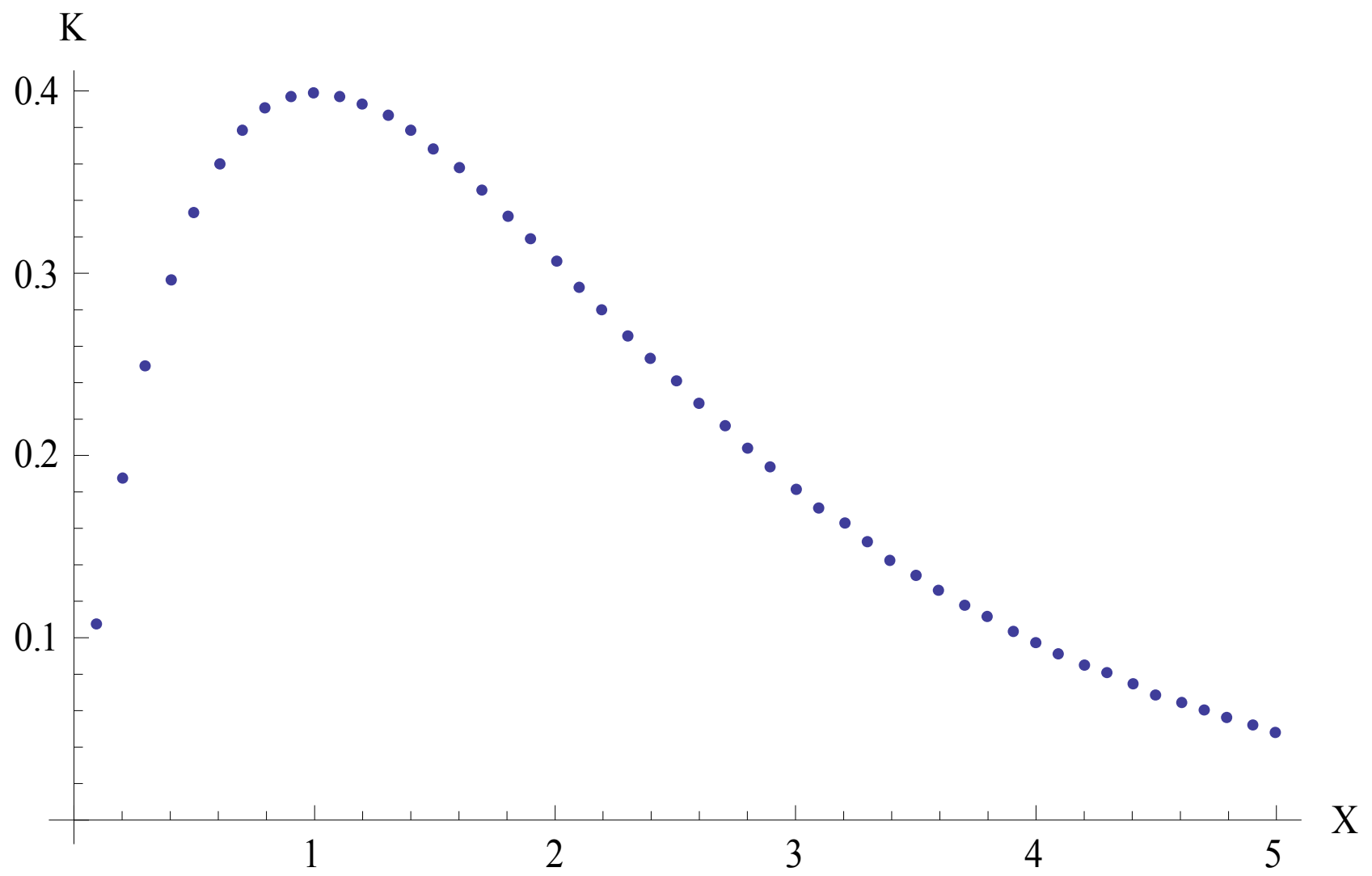

